\title{
Low Prevalence of Pneumocystis pneumonia (PCP) but High Prevalence of Pneumocystis dihydropteroate synthase (dhps) Gene Mutations in HIV-Infected Persons in Uganda
}

\author{
Steve M. Taylor ${ }^{1,2 *}$, Steven R. Meshnick', William Worodria ${ }^{3,4}$, Alfred Andama ${ }^{3,4}$, Adithya Cattamanchi ${ }^{3,5}$, \\ J. Lucian Davis ${ }^{3,5}$, Samuel D. Yoo ${ }^{3,6}$, Patrick Byanyima ${ }^{3}$, Sylvia Kaswabuli ${ }^{3}$, Carol D. Goodman ${ }^{7}$, \\ Laurence Huang ${ }^{3,5,8}$, On behalf of the International HIV-associated Opportunistic Pneumonias \\ (IHOP) Study
}

1 Department of Epidemiology, Gillings School of Global Public Health, University of North Carolina, Chapel Hill, North Carolina, United States of America, 2 Division of Infectious Diseases and International Health, Duke University Medical Center, Durham, North Carolina, United States of America, 3 Makerere University-University of California, San Francisco Research Collaboration, Kampala, Uganda, 4 Department of Medicine, Makerere College of Health Sciences, Mulago Hospital, Kampala, Uganda, 5 Division of Pulmonary and Critical Care Medicine, San Francisco General Hospital, University of California San Francisco, San Francisco, California, United States of America, 6 Health Tutors' College Mulago, Kampala, Uganda, 7 Department of Laboratory Medicine, San Francisco General Hospital, San Francisco, California, United States of America, 8 HIV/AIDS Division, San Francisco General Hospital, University of California San Francisco, San Francisco, California, United States of America

\begin{abstract}
Pneumocystis jirovecii pneumonia (PCP) is an important opportunistic infection in patients infected with HIV, but its burden is incompletely characterized in those areas of sub-Saharan Africa where HIV is prevalent. We explored the prevalence of both PCP in HIV-infected adults admitted with pneumonia to a tertiary-care hospital in Uganda and of putative $P$. jirovecii drug resistance by mutations in fungal dihydropteroate synthase (dhps) and dihydrofolate reductase (dhfr). In 129 consecutive patients with sputum smears negative for mycobacteria, 5 (3.9\%) were diagnosed with PCP by microscopic examination of Giemsa-stained bronchoalveolar lavage fluid. Concordance was $100 \%$ between Giemsa stain and PCR (dhps and dhfr). PCP was more prevalent in patients newly-diagnosed with HIV (11.4\%) than in patients with known HIV (1.1\%; $p=0.007$ ). Mortality at 2 months after discharge was $29 \%$ overall: $28 \%$ among PCP-negative patients, and $60 \%$ (3 of 5 ) among PCP-positive patients. In these 5 fungal isolates and an additional 8 from consecutive cases of PCP, all strains harbored mutant dhps haplotypes; all 13 isolates harbored the P57S mutation in dhps, and 3 (23\%) also harbored the T55A mutation. No non-synonymous dhfr mutations were detected. PCP is an important cause of pneumonia in patients newlydiagnosed with HIV in Uganda, is associated with high mortality, and putative molecular evidence of drug resistance is prevalent. Given the reliability of field diagnosis in our cohort, future studies in sub-Saharan Africa can investigate the clinical impact of these genotypes.
\end{abstract}

Citation: Taylor SM, Meshnick SR, Worodria W, Andama A, Cattamanchi A, et al. (2012) Low Prevalence of Pneumocystis pneumonia (PCP) but High Prevalence of Pneumocystis dihydropteroate synthase (dhps) Gene Mutations in HIV-Infected Persons in Uganda. PLoS ONE 7(11): e49991. doi:10.1371/journal.pone.0049991

Editor: Ana Paula Arez, Instituto de Higiene e Medicina Tropical, Portugal

Received May 15, 2012; Accepted October 19, 2012; Published November 16, 2012

Copyright: (c) 2012 Taylor et al. This is an open-access article distributed under the terms of the Creative Commons Attribution License, which permits unrestricted use, distribution, and reproduction in any medium, provided the original author and source are credited.

Funding: This work was supported by the National Heart, Lung, and Blood Institute [K24 HL087713 and R01 HL090355 to L.H.] and by the National Institute of Allergy and Infectious Diseases [K23 Al080147 to JLD]. The funders had no role in study design, data collection and analysis, decision to publish, or preparation of the manuscript.

Competing Interests: The authors have declared that no competing interests exist.

*E-mail: taylo115@email.unc.edu

- Membership of the International HIV-associated Opportunistic Pneumonias (IHOP) Study is provided in the Acknowledgments.

\section{Introduction}

Pneumocystis jïrovecii pneumonia (PCP) is a major opportunistic infection in people with HIV and AIDS. In the United States and Europe, its incidence has decreased dramatically among HIVinfected patients owing to the twin interventions of antimicrobial prophylaxis of high-risk patients and combination antiretroviral (ARV) therapy. However, in sub-Saharan Africa, access to these interventions remains limited for the 23 million people living with HIV infection; recent estimates indicate that the prevalence of PCP amongst HIV-infected patients with pneumonia may be as high as $27 \%$ in some African countries [1].
PCP prophylaxis decreases the risks of PGP, PCP-related death, and all-cause death amongst patients with AIDS. [2] However, putative $P$. jirovecii drug resistance threatens the durability of PCP prophylaxis with antifolate antibiotics, which are the mainstay of PCP prevention amongst high-risk patients. Antifolate drugs target two enzymes that are necessary for the fungus to synthesize folate dihydropteroate synthase (DHPS) and dihydrofolate reductase (DHFR) - and mutations in the genes encoding these proteins ( $d h p s$ and $d h f r$, respectively) are associated with reduced in vitro drug efficacy. [3,4] Because drug efficacy cannot be directly assessed in the absence of a reliable $P$. jirovecii cultivation system, and because the presence of several mutations is consistently associated with 
exposure to antifolates, [5] these mutations may represent a genetic signature of clinical drug resistance. In the United States and Europe, the prevalence of two of the most common dhps mutations (those at codons 55 and 57) in patients with PCP has been reported between $23-81 \%$ (in the USA) and 7-57\% (in Europe). [6] Estimates of the independent effect of $P$. jirovecii mutants upon clinical outcomes have been conflicting: some studies report their association with increased mortality or antifolate-based treatment failure, [7-10] while others have not [11-13].

Despite the potential high burden of PCP in sub-Saharan Africa, few studies have explored $P$. jirovecii $d h p s$ and $d h f r$ genotypes in HIV-infected patients in this region, and no studies have done so outside of Southern Africa. [14,15] We investigated P. jirovecii among a cohort of HIV-infected patients admitted to Mulago Hospital, a national referral center in Kampala, Uganda. We assessed the prevalence both of PCP amongst patients with pneumonia and of $P$. jirovecii mutations associated with antifolate drug resistance. We hypothesized that $\mathrm{PCP}$ would be common and that mutations would be associated with reported exposure to antifolates.

\section{Methods}

\section{Ethics Statement}

The study was approved by institutional review boards at Mulago Hospital and Makerere University, the University of California, San Francisco, the University of North Carolina at Chapel Hill, and by the Uganda National Council for Science and Technology. All patients provided written informed consent.

\section{Patient Enrollment and Sample Collection}

For this study, $P$. jirovecii molecular testing was employed in two phases in an effort to first assess the prevalence of PCP amongst HIV-infected patients with pneumonia and subsequently to assess the prevalence of mutant $P$. jirovecii genotypes among patients with microscopically-confirmed PCP while also optimizing resources. In phase 1, we tested consecutively-enrolled patients meeting the criteria below between September 2007 and July 2008, to determine the prevalence of PCP at Mulago Hospital and to allow for comparison of Pneumocystis detection by microscopy and PCR. After we found a low prevalence of PCP in phase 1, in phase 2 , we obtained molecular sequence data from only those patients with microscopically-diagnosed PCP between September 2008 and October 2009.

Details of the clinical study have been described previously. [16] Briefly, consecutive patients that were admitted to Mulago Hospital were screened; patients were offered enrollment if they were HIV-positive, had cough $\geq 2$ weeks but $<6$ months, and received a clinical diagnosis of pneumonia. All patients had a chest $\mathrm{x}$-ray and provided two sputum samples for examination for acidfast bacilli (AFB) by direct Ziehl-Neelsen microscopy. Clinical outcome was assessed either in person or by telephone interview at 2 months after discharge.

Patients with sputa negative for AFB were referred for bronchoscopy. Two experienced pulmonologists performed bronchoscopies by completely visualizing central airways and lavaging the radiographically-most-affected lobe of the lung with up to $125 \mathrm{~mL}$ of sterile normal saline. Trained laboratory technicians in the Microbiology Department at Mulago Hospital performed all initial examinations of BAL fluid, including modified Giemsa stain for P. jirovecii; Giemsa-stained BAL fluid was subsequently assessed by a second reader at San Francisco General Hospital who was masked to other clinical and laboratory data. Aliquots of $1-2 \mathrm{~mL}$ of unprocessed BAL fluid were frozen at $-20^{\circ} \mathrm{C}$ for subsequent molecular analyses.

\section{Molecular Methods}

PCR testing was performed at the University of North Carolina by personnel masked to clinical and microscopic diagnoses. BAL specimens were thawed and briefly vortexed. Genomic DNA (gDNA) was extracted from $200 \mu \mathrm{L}$ of each BAL specimen using the EZ1 Virus Mini Kit v2.0 (Qiagen, Valencia, CA). Individual samples were amplified in nested PCR assays targeting the $d h p s$ and $d h f r$ genes of $P$. jirovecii. $[17,18]$ Products were electrophoresed on $1 \%$ agarose gels, with amplification confirmed by the presence of a single band at 330 base pairs (for $d h p s$ ) or at 850 base pairs (for $d h f r)$. Separate work areas for gDNA extraction, reaction set-up, and electrophoresis were maintained to minimize contamination of the PCR testing, and all reaction plates included negative and positive controls with either molecular-grade water or gDNA from a patient with known PCP, respectively.

Samples demonstrating amplification were purified with the QIAquick Gel Extraction Kit (Qiagen). Purified products were bidirectionally sequenced using ABI BigDye Terminator chemistry at the UNC Core Sequencing Facility. Sequences were aligned with Sequencher v4.8 (Gene Codes, Ann Arbor, MI). Wildtype $P$. jirovecii sequences obtained from GenBank were used as referents for alignments: U66282 for dhps and AF090368 for dhfr. Base calls at loci corresponding to codons 55 and 57 of dhps were confirmed by manual inspection of the chromatograms of contigs that were built from both forward and reverse sequencing reads. Aligned sequences were manually inspected both for mixed alleles (defined as peaks $\geq 10 \%$ of primary peak, above baseline) and for mutations at other loci (including those in the sequenced fragment of $d h f r)$; novel mutations were defined as single nucleotide polymorphisms evident in both sequences obtained from the same amplicon at a locus not described above.

\section{Definitions and Statistical Analyses}

Haplotypes for $d h p s$ were assigned based upon codons 55 and 57: "Wildtype" refers to isolates with wildtype alleles at both loci, "single-mutant" to isolates with a wildtype allele at only one locus, and "double-mutant" to isolates with mutant alleles at both loci. For haplotype assignment, mixed alleles were classified as mutant.

We compared demographic and clinical data between patients with and without PCP with the student's t-test or Kruskal-Wallis test for continuous variables, or with the chi-squared test for categorical variables. We compared agreement between PCP diagnosis by modified Giemsa stain and PCR using Cohen's kappa. All statistical analyses were conducted with Stata/IC (version 10, Stata Corp., College Station, TX).

\section{Results}

\section{Prevalence of PCP}

In phase 1 overall, 407 consecutive patients were enrolled and 218 (54\%) were eligible for bronchoscopy; 132 patients $(32 \%)$ ultimately underwent bronchoscopy and BAL. Therefore, 129 patients were investigated in phase 1 (three specimens were missing), and clinical characteristics were similar between those who did and did not undergo bronchoscopy. [19] Mean (SD) age of these 129 patients was 34 years (9.1), and 73 (57\%) were women. Ninety-four patients $(73 \%)$ had been previously diagnosed with HIV. Among these 94 patients, $22(23.4 \%)$ were taking antiretrovirals on admission, and 77 (81\%) reported taking PCP prophylaxis (76 with trimethoprim-sulfamethoxazole, 1 with dapsone). The median (interquartile range) CD4 cell 
count among 128 patients with available values was 74 cells/ $\mathrm{mm}^{3}(17-192)$, and 98 patients $(77 \%)$ had a CD4 cell count below 200 cells $/ \mathrm{mm}^{3}$.

As previously described, the most frequent pulmonary diagnoses in these patients who underwent bronchoscopy were pulmonary tuberculosis (TB), pulmonary cryptococcosis, pulmonary Kaposi sarcoma, and PCP. [20] By microscopic evaluation of modified Giemsa-stained BAL specimens, five patients (3.9\%) were diagnosed with PCP. Two patients were diagnosed with PCP alone, and one patient each was also co-infected with pulmonary Cryptococcus neoformans, culture-positive pulmonary tuberculosis, or culture-negative pulmonary tuberculosis. Four of the five were newly diagnosed with HIV; all five had CD4 cell counts below 50 cells $/ \mathrm{mm}^{3}$, and none were taking antiretrovirals or PCP prophylaxis. Overall mortality at 2 months after discharge was 29\% (38 of 129): $28 \%$ of non-PCP patients had died (35 of 124), compared with $60 \%$ of PCP patients ( 3 of 5; Risk Ratio 2.13; 95\% C.I. $0.98-$ 4.59).

Five patients $(3.9 \%)$ were positive in the both of the PCR assays targeting $d h p s$ and $d h f r$. Agreement between detection by microscopy and PCR was $100 \%(\mathrm{p}<0.001)$. Additionally, agreement between the reader at Mulago Hospital and the second, masked reader at San Francisco General Hospital was also 100\%.

\section{Prevalence of dhps and dhfr Genotypes}

In total, $13 d h p s$ and $d h f r$ sequences were available ( 5 from phase 1 and an additional 8 from phase 2) (Table 1). All 13 isolates harbored the P57S mutation in dhps; in one specimen, the mutation was present in addition to the wildtype allele. Only 3 $(23 \%)$ harbored the T55A mutation; in one specimen, the mutation was present with the wildtype allele.

The single-mutant dhps haplotype was present in 10/13 (77\%) specimens, and the double-mutant $d h p s$ haplotype was present in the remaining three. Thus, there were no wildtype $d h p s$ haplotypes present.

In 12 of 13 specimens dhfr was wildtype across the sequenced loci. One specimen demonstrated a synonymous thymidine to cytosine substitution at base pair 1437 of the reference sequence. Thus, none of the isolates harbored mutant DHFR enzymes.

Table 1. Prevalence of $P$. jirovecii genotypes.

\begin{tabular}{|c|c|}
\hline & No. $(\%)$ of patients $(n=13)$ \\
\hline \multicolumn{2}{|c|}{ dhps mutant codons } \\
\hline T55A & $3(23.1)$ \\
\hline P57S & $13(100)$ \\
\hline \multicolumn{2}{|l|}{ dhps haplotypes } \\
\hline Wildtype & 0 \\
\hline Single mutant & $10(76.9)$ \\
\hline Double mutant & $3(23.1)$ \\
\hline \multicolumn{2}{|l|}{ dhfr haplotypes } \\
\hline Wildtype & $12(92.3)$ \\
\hline Mutant* & $1(7.7)$ \\
\hline \multicolumn{2}{|c|}{$\begin{array}{l}\text { dhps: dihydropteroate synthase; } d h f r \text { : dihydrofolate reductase. Patients with } \\
\text { mixed alleles were classified as mutant. } \\
\text { *The single } \text { dhfr polymorphism encoded a synonymous mutation at base pair } \\
1437 \text { of the reference sequence AF090368. } \\
\text { doi:10.1371/journal.pone.0049991.t001 }\end{array}$} \\
\hline
\end{tabular}

\section{Clinical and Molecular Correlates}

There were no significant differences in the mean age or gender distribution of patients with and without PCP (Table 2). All patients with PCP had a CD4 count $<200$ cells/mm3; median CD4 cell count was lower among patients diagnosed with PCP (12 cells/mm3) than without (88; $\mathrm{p}=0.033$ by Kruskal-Wallis). No PCP patients were taking antiretrovirals on admission.

PCP was significantly more prevalent among the 35 patients newly diagnosed with HIV $(11.4 \%)$ than the 94 patients with previously-diagnosed HIV (1.1\%; $\mathrm{p}=0.007)$. Among the 94 patients with known HIV, PCP was diagnosed in 1 of $17(5.9 \%)$ not taking PCP prophylaxis and 0 of 77 who reported receiving PCP prophylaxis $(\mathrm{p}=0.032)$.

Among the 13 total patients with PCP, the dhps double mutant was present in 1 of 2 patients taking PCP prophylaxis (both with trimethoprim-sulfamethoxazole) and 2 of 11 (18.2\%) not taking prophylaxis $(\mathrm{p}=0.326)$. Only $1 \mathrm{dhfr}$ mutant was identified, this in a patient not taking PCP prophylaxis. The absence of wildtype $d h p s$ genotypes precluded a meaningful assessment of the effect of $d h p s$ genotype upon clinical PCP outcome.

\section{Discussion}

In this cross-sectional study of HIV-infected Ugandans admitted to a tertiary care hospital with pneumonia who underwent bronchoscopy, the prevalence of Pneumocystis pneumonia was low, but all $P$. jirovecii isolates harbored mutations in the $d h p s$ gene. These mutations are putatively associated with antifolate resistance based upon epidemiologic evidence and experience with other pathogens. Additionally, the field diagnosis of PCP using Giemsa staining demonstrated excellent concordance with molecular diagnosis. Because PCP remains an important opportunistic infection in $\mathrm{HIV}$-infected patients that are not receiving PCP prophylaxis, these findings have important implications for the care of patients with AIDS in sub-Saharan Africa.

Our data indicate that $P$. jirovecii is now an uncommon etiology of pneumonia in hospitalized, HIV-infected patients in Kampala, Uganda. Reported prevalences of PCP in sub-Saharan African settings vary widely, including $4 \%$ in the Central African Republic, [21] 5\% in Bujumbura, [22] 5\% in Kigali, [23] 8\% in Dakar, [21] 8\% in Moshi, [24] 27\% in Blantyre, [1] 30\% in Addis Ababa, [25] and 33\% in Harare. [26] Indeed, the prevalence of PCP (diagnosed using an immunofluorescent antibody test) was $39 \%$ among patients admitted to Mulago Hospital and enrolled with similar criteria to ours between 1999 and 2000. [27] Importantly, only $25 \%$ of patients in the earlier cohort reported by Worodria et al. reported receiving cotrimoxazole prior to admission, [27] while in our cohort, $60 \%$ of all patients and $81 \%$ of those who were previously-diagnosed with HIV received PCP prophylaxis. Our study was not designed to assess the efficacy of PCP prophylaxis, but among patients with known HIV, we do note a significant negative association between PCP diagnosis and the receipt of PCP prophylaxis. Therefore, the successful provision of PCP prophylaxis to HIV-infected patients in Kampala that comprised our cohort may have reduced the overall prevalence of PCP.

Despite the low overall PCP prevalence, among patients newlydiagnosed with HIV, the prevalence of PCP was $11.4 \%$, indicating that PCP remains a significant clinical concern in those newlydiagnosed with HIV or not on PCP prophylaxis. Our study highlights the applicability of microscopic examination of modified Giemsa-stained pulmonary specimens for field diagnosis of PCP: Agreement was 100\% between this technique and PCR at the $d h p s$ and $d h f r$ loci. Several studies in high income settings have 
Table 2. Comparison of 129 consecutive HIV-infected patients with and without Pneumocystis pneumonia (PCP).

\begin{tabular}{|c|c|c|c|}
\hline & PCP-positive $(n=5)$ & PCP-negative $(n=124)$ & p-value* \\
\hline Age, yr, mean (SD) & $35.2(8.9)$ & $34.0(9.1)$ & 0.78 \\
\hline Women, no. (\%) & $2(40)$ & $71(57.3)$ & 0.45 \\
\hline CD4 cell count, cells $/ \mu \mathrm{l}$, median (interquartile range) & $12(10-39)$ & $88(22-196)$ & 0.033 \\
\hline CD4 cells $<\mathbf{2 0 0}$ cells $/ \mu \mathrm{l}, \%$ & $5(100)$ & $93(75.6)$ & 0.21 \\
\hline New HIV diagnosis, no. (\%) & $4(80)$ & $31(25)$ & 0.007 \\
\hline Taking PCP prophylaxis**, no. (\%) & $0(0)$ & $77(82.8)$ & 0.03 \\
\hline Receiving antiretrovirals**, no. (\%) & $0(0)$ & $22(23.7)$ & 0.58 \\
\hline \multicolumn{4}{|l|}{ Positive PCR assay, no. (\%) } \\
\hline dhps & $5(100)$ & 0 & NA \\
\hline dhfr & $5(100)$ & 0 & NA \\
\hline
\end{tabular}

HIV: human immunodeficiency virus; PCR: polymerase chain reaction; dhps: dihydropteroate synthase; dhfr: dihydrofolate reductase; NA: not applicable. *Determined by the student's t-test or Kruskal-Wallis test for continuous variables, or the chi-squared test for categorical variables.

**Among 94 patients with previously-diagnosed HIV.

doi:10.1371/journal.pone.0049991.t002

described the performance characteristics of the modified Giemsa method in comparison with staining with Grocott's methenamine silver and toluidine blue-O or direct and indirect immunofluorescence staining, $[28,29]$ but its performance has not been formally reported from low- and middle-income settings, where the burdens of AIDS and PCP are greatest. Though immunofluorescent staining is most commonly employed for diagnosis in high income settings, its expense precludes its wide use in many low- and middle-income settings. Our data suggest that the less-expensive modified Giemsa stain is a viable option for reliable PCP diagnosis in settings with high prevalences of HIV in settings such as subSaharan Africa.

All patients in our study harbored mutant $P$. jurovecii dhps isolates. Though this may derive from a small sample size, this is notable both because it exceeds prevalences reported in other regions and because most patients individually denied prior exposure to PCP prophylaxis with antifolate drugs, which is commonly associated with the presence of $P$. jirovecii mutations. [5] Prevalences of mutant $P$. jirovecii dhps genotypes vary geographically, [6,30] and are generally highest in high income settings. [31] Fewer reports have reported prevalences from sub-Saharan Africa, though $56 \%$ of isolates from South Africa in 2006 harbored mutant dhps haplotypes. [14] The high prevalence in our study despite the lack of individual antifolate PCP prophylaxis may derive from two nonexclusive processes: interhuman transmission of mutant strains or population-level selective pressure exerted by antifolate use for other indications. Interhuman transmission of mutant strains has been inferred from ecological studies of mutant genotypes in which geographic residence was associated with genotype. [30] Though the factors that mediate the transmission of $P$. jirovecii remain incompletely understood, two additional findings in Uganda argue against this explanation. Specimens with mutant $d h p s$ haplotypes were not clonal, as we detected 3 different haplotypes of the $P$. jirovecii mitochondrial large subunit of ribosomal DNA in specimens with identical mutant dhps haplotypes. [16] Additionally, $P$. jirovecii colonization - one putative reservoir for interhuman transmission - was rare in our cohort, with only $6 \%$ of patients with non-Pneumocystis pneumonia harboring $P$. jirovecii DNA as measured by a different genomic marker that amplifies a multicopy gene as opposed to the single-copy dhps and dhfr genes. [16] Taken together, the non-clonality of mutant strains and the low prevalence of $P$. jirovecii colonization suggest that interhuman transmission was not primarily responsible for the high prevalence of mutant Pneumocystis.

Notably, all patients in our study harbored Pneumocystis strains with the dhps P57S mutation, and this near-fixation of the P57S mutation suggests significant population-level selective pressure. Notably, the pattern of mutant haplotypes - with a preponderance of single-mutant P57S haplotypes - is similar to that observed in a study from France, wherein a high prevalence of these haplotypes was associated with the use of sulfadoxine-pyrimethamine (SP, or Fansidar) as prophylaxis as compared with the use of trimethoprim-sulfamethoxazole (co-trimoxazole). [32] SP use is prevalent is Uganda: until recently, SP was recommended first-line therapy for malaria, and at the time of this study was still commonly used for treatment of suspected malaria. [33] Taken together, the previous literature linking SP and the P57S mutation, the high prevalence of the P57S mutation in our study, and the widespread use of SP in Uganda during this period, suggest that sulfadoxine may differentially select for the P57S mutation in the dhps gene of $P$. jirovecii.

We observed no non-synonymous mutations in the sequenced segment of $d h f r$. Mutations in $P$. jirovecii $d h f r$ vary greatly in prevalence and allele between published studies, [12,18,34] and in some settings non-synonymous mutations have been associated with the receipt of pyrimethamine-containing PCP prophylaxis. [35] Though we failed to detect any such mutations, the small sample size of $d h f r$ haplotypes in our cohort precludes inferences of selection pressure on P. jirovecii dhfr haplotypes in Uganda.

Our study has several limitations. The high prevalence of PCP prophylaxis use likely reduced the prevalence of PCP in our cohort and may fetter comparison with other settings where PCP prevalence is higher. Additionally, though concordance between microscopic and molecular diagnosis was excellent, the use of BAL specimens in our study may limit the generalizability of this observation. Furthermore, a number of eligible patients did not undergo bronchoscopy owing to patient refusal, death, or the establishment of an alternate firm diagnosis, [20] and so the true prevalence of PCP cannot be determined conclusively. Clinical data that are commonly employed to gauge the likelihood of PCP - including serum lactate dehydrogenase, arterial blood gas, chest radiograph - were not uniformly obtained, precluding assessment of their relationship to microscopically-confirmed PCP in this setting. Finally, the limited number of fungal genotypes prevented 
a fuller characterization of the relationship between clinical and molecular data and continued study is warranted.

Our study of pneumonia amongst HIV-infected Ugandans highlights the need to consider PCP as an etiology of pneumonia in sputum AFB smear-negative patients with newly-diagnosed $\mathrm{HIV}$ and those not on PCP prophylaxis in Uganda, and describes a clinically- and cost-effective method for field diagnosis. Additionally, we describe the near-fixation of a specific $P$. jirovecii dhps mutation, which may be associated with patterns of sulfa antibiotic use. Given the high prevalence of this haplotype which is associated with drug resistance and the large population at risk of PCP in sub-Saharan Africa, future studies are required to more definitively characterize the clinical impact of these mutations.

\section{References}

1. Hartung TK, Chimbayo D, van Oosterhout JJ, Chikaonda T, van Doornum GJ, et al. (2011) Etiology of suspected pneumonia in adults admitted to a highdependency unit in Blantyre, Malawi. Am J Trop Med Hyg 85: 105-112.

2. Ioannidis JP, Cappelleri JC, Skolnik PR, Lau J, Sacks HS (1996) A meta-analysis of the relative efficacy and toxicity of Pneumocystis carinii prophylactic regimens. Arch Intern Med 156: 177-188.

3. Iliades P, Meshnick SR, Macreadie IG (2005) Mutations in the Pneumocystis jirovecii DHPS gene confer cross-resistance to sulfa drugs. Antimicrob Agents Chemother 49: 741-748.

4. Meneau I, Sanglard D, Bille J, Hauser PM (2004) Pneumocystis jiroveci dihydropteroate synthase polymorphisms confer resistance to sulfadoxine and sulfanilamide in Saccharomyces cerevisiae. Antimicrob Agents Chemother 48: 2610-2616.

5. Stein CR, Poole C, Kazanjian P, Meshnick SR (2004) Sulfa use, dihydropteroate synthase mutations, and Pneumocystis jirovecii pneumonia. Emerg Infect Dis 10: $1760-1765$

6. Matos O, Esteves F (2010) Epidemiology and clinical relevance of Pneumocystis jirovecii Frenkel, 1976 dihydropteroate synthase gene mutations. Parasite 17: 219-232.

7. Helweg-Larsen J, Benfield TL, Eugen-Olsen J, Lundgren JD, Lundgren B (1999) Effects of mutations in Pneumocystis carinii dihydropteroate synthase gene on outcome of AIDS-associated P. carinii pneumonia. Lancet 354: 1347-1351.

8. Kazanjian P, Armstrong W, Hossler PA, Burman W, Richardson J, et al. (2000) Pneumocystis carinii mutations are associated with duration of sulfa or sulfone prophylaxis exposure in AIDS patients. J Infect Dis 182: 551-557.

9. van Hal SJ, Gilgado F, Doyle T, Barratt J, Stark D, et al. (2009) Clinical significance and phylogenetic relationship of novel Australian Pneumocystis jirovecii genotypes. J Clin Microbiol 47: 1818-1823.

10. Valerio A, Tronconi E, Mazza F, Fantoni G, Atzori C, et al. (2007) Genotyping of Pneumocystis jiroveci pneumonia in Italian AIDS patients. Clinical outcome is influenced by dihydropteroate synthase and not by internal transcribed spacer genotype. J Acquir Immune Defic Syndr 45: 521-528.

11. Navin TR, Beard CB, Huang L, del Rio C, Lee S, et al. (2001) Effect of mutations in Pneumocystis carinii dihydropteroate synthase gene on outcome of $\mathrm{P}$ carinii pneumonia in patients with HIV-1: a prospective study. Lancet 358 : $545-549$.

12. Ma L, Borio L, Masur H, Kovacs JA (1999) Pneumocystis carinii dihydropteroate synthase but not dihydrofolate reductase gene mutations correlate with prior trimethoprim-sulfamethoxazole or dapsone use. J Infect Dis 180: 1969-1978.

13. Ma L, Kovacs JA, Cargnel A, Valerio A, Fantoni G, et al. (2002) Mutations in the dihydropteroate synthase gene of human-derived Pneumocystis carinii isolates from Italy are infrequent but correlate with prior sulfa prophylaxis. J Infect Dis 185: 1530-1532.

14. Dini L, du Plessis M, Frean J, Fernandez V (2010) High prevalence of dihydropteroate synthase mutations in Pneumocystis jirovecii isolated from patients with Pneumocystis pneumonia in South Africa. J Clin Microbiol 48: 2016-2021.

15. Zar HJ, Alvarez-Martinez MJ, Harrison A, Meshnick SR (2004) Prevalence of dihydropteroate synthase mutants in HIV-infected South African children with Pneumocystis jiroveci pneumonia. Clin Infect Dis 39: 1047-1051.

16. Taylor SM, Meshnick SR, Worodria W, Andama A, Davis JL, et al. (2011) Low prevalence of Pneumocystis jirovecii lung colonization in Ugandan HIV-infected patients hospitalized with non-Pneumocystis pneumonia. Diagnostic Microbiology \& Infectious Disease.

17. Alvarez-Mart ̃̃-nez Mr, MirÃ3 J, Valls ME, Moreno An, Rivas P, et al. (2006) Sensitivity and specificity of nested and real-time PCR for the detection of Pneumocystis jiroveci in clinical specimens. Diagnostic microbiology and infectious disease 56: 153-160.

\section{Acknowledgments}

We thank Florence Nankya, John Kiidha, Rachel Kyeyune, Catherine Nabakiibi, Nelson Kalema, and Margaret Awor for their efforts and the administration of Mulago Hospital for their support of our research and patient care efforts. Saskia den Boon was critical to the conduct of the study. Ultimately, we are indebted to the patients who participated in the study.

\section{Author Contributions}

Conceived and designed the experiments: SMT SRM WW SDY LH. Performed the experiments: SMT WW SDY PB SK CDG. Analyzed the data: SMT WW AA JLD AC SDY LH. Contributed reagents/materials/ analysis tools: WW AA PB SK CDG. Wrote the paper: SMT SRM LH.

18. Costa MC, Esteves F, Antunes F, Matos O (2006) Genetic characterization of the dihydrofolate reductase gene of Pneumocystis jirovecii isolates from Portugal. J Antimicrob Chemother 58: 1246-1249.

19. Deok-jong Yoo S, Worodria W, Davis JL, Cattamanchi A, den Boon S, et al (2010) The prevalence and clinical course of HIV-associated pulmonary cryptococcosis in Uganda. J Acquir Immune Defic Syndr 54: 269-274.

20. Worodria W, Davis JL, Cattamanchi A, Andama A, den Boon S, et al. (2010) Bronchoscopy is useful for diagnosing smear-negative tuberculosis in HIVinfected patients. Eur Respir J 36: 446-448.

21. Vray M, Germani Y, Chan S, Duc NH, Sar B, et al. (2008) Clinical features and etiology of pneumonia in acid-fast bacillus sputum smear-negative HIV-infected patients hospitalized in Asia and Africa. AIDS 22: 1323-1332.

22. Kamanfu G, Mlika-Cabanne N, Girard PM, Nimubona S, Mpfizi B, et al. (1993) Pulmonary complications of human immunodeficiency virus infection in Bujumbura, Burundi. Am Rev Respir Dis 147: 658-663.

23. Batungwanayo J, Taelman H, Lucas S, Bogaerts J, Alard D, et al. (1994) Pulmonary disease associated with the human immunodeficiency virus in Kigali, Rwanda. A fiberoptic bronchoscopic study of 111 cases of undetermined etiology. Am J Respir Crit Care Med 149: 1591-1596.

24. Kibiki GS, Beckers P, Mulder B, Arens T, Mueller A, et al. (2007) Aetiology and presentation of HIV/AIDS-associated pulmonary infections in patients presenting for bronchoscopy at a referral hospital in northern Tanzania. East Afr Med J 84: 420-428.

25. Aderaye G, Bruchfeld J, Aseffa G, Nigussie Y, Melaku K, et al. (2007) Pneumocystis jiroveci pneumonia and other pulmonary infections in TB smearnegative HIV-positive patients with atypical chest X-ray in Ethiopia. Scandinavian journal of infectious diseases 39: 1045-1053.

26. Malin AS, Gwanzura LK, Klein S, Robertson VJ, Musvaire P, et al. (1995) Pneumocystis carinii pneumonia in Zimbabwe. Lancet 346: 1258-1261.

27. Worodria W, Okot-Nwang M, Yoo SD, Aisu T (2003) Causes of lower respiratory infection in $\mathrm{HIV}$-infected Ugandan adults who are sputum AFB smear-negative. Int J Tuberc Lung Dis 7: 117-123.

28. Ng VL, Yajko DM, McPhaul LW, Gartner I, Byford B, et al. (1990) Evaluation of an indirect fluorescent-antibody stain for detection of Pneumocystis carinii in respiratory specimens. J Clin Microbiol 28: 975-979.

29. Cregan P, Yamamoto A, Lum A, VanDerHeide T, MacDonald M, et al. (1990) Comparison of four methods for rapid detection of Pneumocystis carinii in respiratory specimens. J Clin Microbiol 28: 2432-2436.

30. Huang L, Beard CB, Creasman J, Levy D, Duchin JS, et al. (2000) Sulfa or sulfone prophylaxis and geographic region predict mutations in the Pneumocystis carinii dihydropteroate synthase gene. J Infect Dis 182: 1192-1198.

31. Crothers K, Beard CB, Turner J, Groner G, Fox M, et al. (2005) Severity and outcome of HIV-associated Pneumocystis pneumonia containing Pneumocystis jirovecii dihydropteroate synthase gene mutations. AIDS 19: 801-805.

32. Hauser PM, Nahimana A, Taffe P, Weber R, Francioli P, et al. (2010) Interhuman transmission as a potential key parameter for geographical variation in the prevalence of Pneumocystis jirovecii dihydropteroate synthase mutations. Clin Infect Dis 51: e28-33.

33. Ndyomugyenyi R, Magnussen P, Clarke S, Ndyomugyenyi R, Magnussen P, et al. (2007) Diagnosis and treatment of malaria in peripheral health facilities in Uganda: findings from an area of low transmission in south-western Uganda. Malaria Journal 6: 39.

34. Siripattanapipong S, Leelayoova S, Mungthin M, Worapong J, Tan-Ariya P (2008) Study of DHPS and DHFR genes of Pneumocystis jirovecii in Thai HIVinfected patients. Med Mycol 46: 389-392.

35. Nahimana A, Rabodonirina M, Bille J, Francioli P, Hauser PM (2004) Mutations of Pneumocystis jirovecii dihydrofolate reductase associated with failure of prophylaxis. Antimicrob Agents Chemother 48: 4301-4305. 